\title{
Glomerulonefritis rápidamente progresiva mediada por inmunocomplejos (tipo II). Revisión de la literatura
}

\section{Immune complex-mediated rapidly progressive glomerulonephritis (type II). Literature review}

John Fredy Nieto-Ríos', Miguel Bernardo Giraldo-Serna², Camilo Andrés GarcíaPrada $^{3}$, Andrés Felipe Lotero-Cadavid ${ }^{4}$, Lina María Serna-Higuita ${ }^{5}$, Luis F. Arias ${ }^{6}$

Resumen. La glomerulonefritis rápidamente progresiva mediada por complejos inmunes (GMNRP II) es un síndrome clínico caracterizado por el rápido deterioro de la función renal asociado a hematuria, edemas y oliguria. Histológicamente se manifiesta como una glomerulonefritis crescéntica, con la presencia de depósitos granulares en la inmunofluorescencia. Aunque es una enfermedad rara, es grave y puede evolucionar a una enfermedad renal crónica, por lo cual es fundamental su identificación temprana. A continuación, se presenta una revisión sobre este tipo de glomerulonefritis, con énfasis en su etiología y en las opciones terapéuticas existentes en la actualidad.

Palabras clave: glomerulonefritis, complejos inmunes, nefropatía por inmunoglobulina A, púrpura de Schönlein-Henoch, insuficiencia renal terminal, biopsia, esteroides.

Abstract. Rapidly progressive immune complex-mediated glomerulonephritis (RPGNMN II) is a clinical syndrome characterized by severe deterioration of renal function associated with hematuria, edema, and oliguria. It is histologically characterized as a crescentic glomerulonephritis, with the presence of granular deposits on immunofluorescence. Although it is a rare condition, it is a potentially serious disease that may progress to chronic renal disease, therefore its early identification

\footnotetext{
1 Médico, Especialista en Medicina Interna, Especialista en Nefrología, Hospital Pablo Tobón Uribe, Universidad de Antioquia. Medellín, Colombia.

2 Médico, Residente de Medicina Interna, Universidad de Antioquia. Medellín Colombia.

${ }^{3}$ Médico, Especialista en Medicina Interna, Especialista en Nefrología, Hospital Universitario San Vicente Fundación, IPS Sura. Medellín, Colombia.

${ }^{4}$ Médico, Residente de Medicina Interna, Universidad Pontificia Bolivariana. Medellín, Colombia.

${ }^{5}$ Médica, Especialista en Pediatría, Especialista en Nefrología Pediátrica, MSc en Epidemiología. Docente e Investigadora Asociada, Universidad de Tübingen. Tübingen, Alemania. E-mail: linasernahiguita@gmail.com.

${ }^{6}$ Médico, Especialista en Patología, PhD en Medicina. Docente, Universidad de Antioquia. Medellín, Colombia.

Conflicto de interés: los autores declaran que no tienen conflicto de interés.

Medicina \& Laboratorio 2021;25:569-580. https://doi.org/10.36384/01232576.449.

Recibido el 12 de mayo de 2021; aceptado el 24 mayo de 2021. Editora Médica Colombiana S.A., $2021^{\circ}$.
} 
is essential. Here we present a review of this form of glomerulonephritis, with emphasis on its etiology and the currently available therapeutic options.

Keywords: glomerulonephritis, immune complexes, IgA nephropathy, Henoch-Schönlein purpura, end stage renal disease, biopsy, steroids.

\section{Introducción}

La glomerulonefritis se caracteriza por la inflamación o daño de una o varias estructuras de los glomérulos; puede ser aguda, subaguda o crónica de acuerdo al tiempo en que se produce el daño glomerular. Se manifiesta por hematuria glomerular y/o proteinuria, con o sin deterioro de la función renal. Puede estar asociada a causas primarias o secundarias (infecciosas, autoinmunes, neoplásicas, metabólicas, hematológicas, tóxicas, etc.) [1].

La glomerulonefritis rápidamente progresiva (GMNRP) es la máxima expresión de daño glomerular, donde hay ruptura de la membrana basal glomerular y proliferación extracapilar. Puede definirse como un síndrome clínico, caracterizado por el rápido deterioro de la función renal en un período de días, semanas o meses, asociado con otros hallazgos típicos como hematuria, edemas y oliguria; la hematuria es de origen glomerular y se caracteriza por la presencia de eritrocitos dismórficos, acantocitos, cilindros celulares y proteinuria [1-3]. Por la presentación clínica, muchas veces es indiferenciable del síndrome nefrítico, pero es la biopsia renal la que por el hallazgo de medias lunas, finalmente establece el diagnóstico.

Histológicamente, la GMNRP se correlaciona con la glomerulonefritis crescéntica (en semiluna), la cual se caracteriza por el compromiso glome- rular inflamatorio grave, resultado de la ruptura del capilar glomerular con la consecuente proliferación de los diferentes tipos de células glomerulares, lo cual conduce a la formación de semilunas, siendo la definición patológica de glomerulonefritis crescéntica, la presencia de más de $50 \%$ de semilunas en una muestra de biopsia renal representativa, es decir, que al menos tenga 10 glomérulos [1-7]. Este punto de corte se escoge debido a que se han realizado estudios en los cuales se ha evidenciado que los pacientes con un compromiso menor a este porcentaje, tienen un curso más indolente de la enfermedad y un mejor pronóstico a largo plazo [8].

Desde hace muchos años se ha tratado de clasificar la GMNRP dependiendo de las características clínicas e histológicas de la misma, así como de la posible etiología del cuadro [1-3,9]. De esta manera, en la actualidad se puede dividir en cuatro grandes grupos:

- Tipo I: asociada con anticuerpos anti membrana basal glomerular, la cual se caracteriza histológicamente por la presencia de depósitos lineales de lgG.

- Tipo II: mediada por complejos inmunes, con la presencia de depósitos granulares en la inmunofluorescencia.

- Tipo III: pauci-inmune, en la cual no hay evidencia de depósitos inmu- 
nes en la inmunohistoquímica, pero se caracteriza por la presencia de anticuerpos anticitoplasma de neutrófilo (ANCA).

- Tipo IV: caracterizada por la presencia de anticuerpos ANCA y anti membrana basal glomerular.

A continuación, se hará énfasis en la glomerulonefritis rápidamente progresiva tipo II, la cual, como se ha mencionado previamente, es mediada por complejos inmunes.

\section{Epidemiología}

La GMNRP tipo II es una enfermedad rara con una prevalencia baja, pero con una presentación clínica grave que puede llevar a la enfermedad renal crónica avanzada y a la muerte del paciente [10]. Sin embargo, se debe mencionar que aproximadamente entre el $2 \%$ al $7 \%$ de las biopsias renales que se realizan, tienen como hallazgo histopatológico la glomerulonefritis crescéntica, pero con una menor proporción de pacientes que, a pesar de tener la clínica y los hallazgos histológicos, desarrollan enfermedad renal crónica [2,11].

Cuando se han realizado estudios tratando de separar cada uno de los tipos de GMNRP, se ha encontrado que la más común de ellas es la de tipo III o pauci-inmune, con un $60 \%$ de los casos en una de las series más significativas; seguida por la tipo II (motivo de nuestra revisión), la cual representa un 24\% de los casos en dicha serie; y, la tipo I, que explica solamente el $15 \%$ de los casos [9]. Además, se debe considerar la edad de los pacientes que presentan el cuadro, pues dependiendo de la misma, habrá unas etiologías más prevalentes como explicación de la GMNRP. Un ejemplo de esto es el grupo de los pa- cientes pediátricos, en quienes la causa más común de GMNRP es la nefropatía por IgA [12]. Otro grupo etario que se debe tener presente en este sentido, es el de los pacientes mayores de 60 años, en quienes la causa más común es la GMNRP pauci-inmune, relacionada con anticuerpos ANCA [10,13].

En un estudio publicado en 2019 por Wu y colaboradores [14], el $22 \%$ de los casos fueron explicados por GMNRP tipo I, mientras que el 78\% fueron explicados por los tipos II y III. Cuando se hizo un análisis de los pacientes con GMNRP tipo II en dicho estudio, se evidenció que la nefropatía por IgA era la más prevalente, con un $42 \%$ de los casos, seguida por la nefritis lúpica con un $21 \%$, la glomerulonefritis por púrpura de Schönlein-Henoch con $10 \%$, la nefritis asociada a virus de la hepatitis B con 5\%, la idiopática con $5 \%$, y otras con el $17 \%$; esto es consistente con otros estudios previos [15]. Otras causas menos frecuentes de GMNRP tipo II, son la glomerulonefritis asociada a infección, la membranosa y la membranoproliferativa.

\section{Fisiopatología}

El mecanismo patogénico en la GMNRP tipo II, inicia con el depósito o con la formación de complejos inmunes y de complemento en los glomérulos. Este proceso patológico termina conduciendo a la ruptura de la membrana basal glomerular [10], y esta pérdida de integridad de la membrana permite el paso de sustancias proinflamatorias (fibrina, moléculas de adhesión, citocinas, factores de crecimiento, factores procoagulantes) y monocitos hacia la cápsula de Bowman [16]. Una vez que estos productos se encuentran en el ovillo glomerular, se da un proceso de proliferación celular de esos monocitos y de los demás componentes de los 
glomérulos (células mesangiales, células epiteliales, podocitos, células dendríticas y linfocitos T), y un aumento de todas las sustancias proinflamatorias, lo que lleva a la formación de semilunas; estas a su vez, tienden a organizarse mediante la producción de colágeno y la infiltración de fibroblastos. Todo lo cual conduce a la fibrosis y esclerosis glomerular, y a la consecuente disfunción del glomérulo [17].

De acuerdo con la etiología específica del cuadro, las semilunas pueden tener un predominio celular, fibrocelular o fibroso, lo cual le confiere mejor o peor pronóstico a la enfermedad. En ese sentido, cuando existe un mayor componente celular, parece haber un mejor pronóstico que cuando el componente es mixto o fibroso, pues este último se relaciona con un proceso patológico más avanzado. Otro factor pronóstico importante es la integridad de la cápsula de Bowman, siendo más común la progresión hacia semilunas fibrosas cuando hay ruptura de la misma, lo que lleva a una acumulación más prominente de fibroblastos y macrófagos [18,19].

\section{Cuadro clínico}

El espectro clínico de la GMNRP es muy variable, pudiéndose presentar como un cuadro leve e insidioso, donde los únicos síntomas podrían ser astenia y adinamia, con la presencia de edemas leves o moderados, o ir hasta un cuadro clínico súbito con la presentación típica de síndrome nefrítico (hematuria, oliguria/anuria, hipertensión, edemas, proteinuria subnefrótica), el cual puede poner en peligro la vida si no se realiza una intervención de manera inmediata. Frecuentemente, el paciente presenta al momento del diagnóstico un gran deterioro de la función renal con necesidad de terapia de reemplazo renal; el cuadro también puede presentarse ocasionalmente con síndrome nefrótico (proteinuria mayor de 3,5 g, edemas, hipoalbuminemia e hipercolesterolemia), pero con gran compromiso de la función renal $[2,8,20,21]$. Adicionalmente, puede haber compromiso pulmonar grave con hemorragia alveolar, o afección de otros sistemas de acuerdo a la patología de base que tenga el paciente.

En el caso particular de la GMNRP tipo II, el cuadro clínico puede variar un poco, dependiendo de la etiología específica de la misma. Esto permite tener algunos indicios sobre aquella enfermedad que llevó al depósito de complejos inmunes, y por lo tanto al desarrollo de la glomerulonefritis [22]; a continuación, se describen algunas de ellas:

- Glomerulonefritis postestreptocócica: el cuadro de GMNRP ocurre en un pequeño porcentaje de los casos, presentándose en menos del $0,5 \%$ de los mismos [23]. Es más frecuente en niños, pero no hay edad en la que no pueda presentarse la enfermedad. Hay un período de latencia entre el inicio de la infección y el desarrollo de la GMNRP, que es en promedio de 1 a 2 semanas para la faringitis, y de 3 a 6 semanas para las infecciones de la piel. Usualmente en esta entidad se encuentran niveles de complemento C3 bajos $y$ anticuerpos antiestreptolisina $O$ (AELO) positivos. También se puede presentar con otro tipo de infecciones donde el período de latencia no es tan evidente, razón por la cual en estos casos se denomina glomerulonefritis asociada a infección [24-27].

- Nefropatía por IgA: en esta entidad, solo del $5 \%$ al $10 \%$ de los casos se presentan como GMNRP. Clínicamente se caracteriza por hematuria glomerular, asociada al rápido de- 
terioro de la función renal, además, en algunos pacientes se puede presentar como hipertensión maligna, lo cual refleja un largo tiempo de evolución de la enfermedad [28]. En algunos casos, la nefropatía por lgA es disparada por episodios de infección del tracto respiratorio y puede manifestarse con dolor en los flancos durante los episodios agudos; en estos casos, la GMNRP ocurre en la primera semana de la infección (sinfaringítica) $[29,30]$.

- Vasculitis por IgA (púrpura de Schönlein-Henoch): la enfermedad tiene una tétrada clásica, la cual consiste en la presencia de púrpura palpable (sin trombocitopenia ni coagulopatía), artralgias/artritis, dolor abdominal y glomerulonefritis (con compromiso similar a la nefropatía por lgA). Esta última se puede presentar entre un $24 \%$ a $50 \%$ de los casos, siendo más común en jóvenes y adultos, manifestándose como un síndrome nefrítico que en pocos casos puede evolucionar a una GMNRP [29-34].

- Nefritis lúpica: cuando esta es la causa de la GMNRP, el paciente puede presentar otras manifestaciones de la enfermedad tan variadas como compromiso de piel o mucosas, articular, de serosas, hematológico, pulmonar, y del sistema nervioso central, entre otros [6].

\section{Diagnóstico}

Teniendo en cuenta que el cuadro de presentación puede ser similar al de otras formas de GMNRP, ante la presencia de un paciente con sospecha de la enfermedad, se debe hacer un enfoque diagnóstico general, teniendo en cuenta todas las posibles etiologías y no solo las del tipo II. En este sentido, la historia clínica y el examen físico son fundamentales, pues permiten orientar la sospecha diagnóstica hacia alguna etiología en particular.

El diagnóstico se puede establecer bien sea frente a un paciente con un cuadro de glomerulonefritis típico, con hematuria, edemas, oliguria e hipertensión (síndrome nefrítico clásico), con otras manifestaciones de enfermedad glomerular como proteinuria, acompañada de hipoalbuminemia, con la presentación aislada de cada uno de los hallazgos anteriores, o como una alteración de las pruebas de función renal en un paciente previamente sano. Otras veces, el cuadro es multisistémico, sumándose al compromiso renal el compromiso pulmonar, manifestado por hemoptisis asociada a hemorragia alveolar, que puede terminar en falla respiratoria asociada y anemia severa; otras manifestaciones frecuentes son la fiebre, lesiones purpúricas en piel, artritis, afección de senos paranasales, compromiso neurológico, etc.

Ante cualquier paciente con sospecha de la enfermedad, se deben realizar los siguientes paraclínicos básicos: hemograma, creatinina, nitrógeno ureico en sangre, ionograma completo, gases arteriales, uroanálisis, proteinuria en orina de 24 horas, albúmina y ecografía renal. Ante una alta sospecha de la enfermedad o características clínicas de la misma, siempre se debe considerar la biopsia renal. Además, hay algunos paraclínicos más especializados, los cuales se deben solicitar de acuerdo a la sospecha clínica, aunque algunos artículos y documentos de revisión del tema, sugieren su realización en todos los casos; entre ellos, ANA, ENA, antiDNA, C3, C4, ANCA (ELISA para PR3 y MPO), anticuerpos anti-MBG, VIH, VHB, $\mathrm{VHC}$, electroforesis de proteínas, crioglobulinas, hemocultivos y ecocardio- 
grama. Cada uno de estos guiará hacia un diagnóstico etiológico específico.

La biopsia renal es el estándar de oro para el diagnóstico de la GMNRP; el hallazgo característico es la presencia de semilunas en más del $50 \%$ de los glomérulos. En algunos casos, hay además proliferación endocapilar y mesangial asociadas; la inmunofluorescencia en la biopsia es la que aclara el diagnóstico. En la GMNRP tipo II son evidentes los depósitos granulares de una o varias inmunoglobulinas y/o del complemento (IgG, IgA, IgM, C3 y C1 q), dependiendo de la etiología específica. Por ejemplo, en la glomerulonefritis por IgA, la inmunofluorescencia será positiva fuertemente para IgA o muchas veces codominante con C3; en la nefritis lúpica habrá un "full house" porque la inmunofluorescencia será positiva para todas las inmunoglobulinas y complemento; en la postestreptocócica será positiva para C3 principalmente, y puede estar acompañado de $\lg G$ (figuras 1 y 2). Como un hallazgo interesante en los pacientes con GMNRP tipo II, hay menor porcentaje de esclerosis global y fibrosis, y menor necrosis y/o atrofia tubular que en los otros tipos de GMNRP.

De esta forma, siempre que se tenga un paciente con sospecha de GMNRP, se debe realizar una serie de estudios diagnósticos dirigidos, los cuales, interpretados en el contexto de una excelente historia clínica y un buen examen físico, pueden ofrecer las herramientas para llegar al diagnóstico etiológico de la misma, y de esta manera realizar un tratamiento oportuno y adecuado.

\section{Tratamiento}

En el tratamiento general se debe determinar si el paciente se encuentra en urgencia dialítica o en una emergencia pulmonar, con necesidad de asegurar la vía aérea (hemorragia alveolar); de ahí la importancia de manejar estos pacientes en unidades de cuidados especiales o cuidados intensivos para monitoreo estricto y tratamiento multidisciplinario. En esta sección se discutirá el manejo inmunosupresor de la GMNRP.

Lo primero a mencionar, en este apartado de tratamiento, es que los pacientes que no lo reciben, terminan progresando a enfermedad renal crónica en estadio 5 con necesidad de terapia de reemplazo renal indefinidamente, en un período comprendido entre unas semanas a unos pocos meses, o lo que es peor, presentando un compromiso multisistémico que puede terminar en la muerte del paciente, la mayoría de las veces por hemorragia alveolar [8]. Por lo tanto, uno de los pilares del tratamiento es el inicio rápido del mismo, con el fin de detener la progresión de la enfermedad, evitar el deterioro irreversible de la función renal y frenar el compromiso multisistémico.

Cuando se descarta una infección grave (por ejemplo, endocarditis, nefritis por shunt, abscesos viscerales, etc.), la primera opción de tratamiento en estos pacientes es el inicio de terapia inmunosupresora agresiva, con esteroides en pulsos por vía parenteral y luego oral a dosis altas, asociada a un medicamento citotóxico, siendo la ciclofosfamida la primera opción, pero teniendo otras opciones disponibles [1,3,9,35]. A continuación, se proponen unos esquemas basados en la literatura:

- Pulsos de metilprednisolona de $500 \mathrm{mg}$ a $1.000 \mathrm{mg}$ intravenosos, cada día por un período de 3 días, seguidos de prednisolona vía oral a dosis de $1 \mathrm{mg} / \mathrm{kg} /$ día (máximo 80 $\mathrm{mg})$, la cual se debe mantener por un espacio de 1 a 2 meses, para lue- 


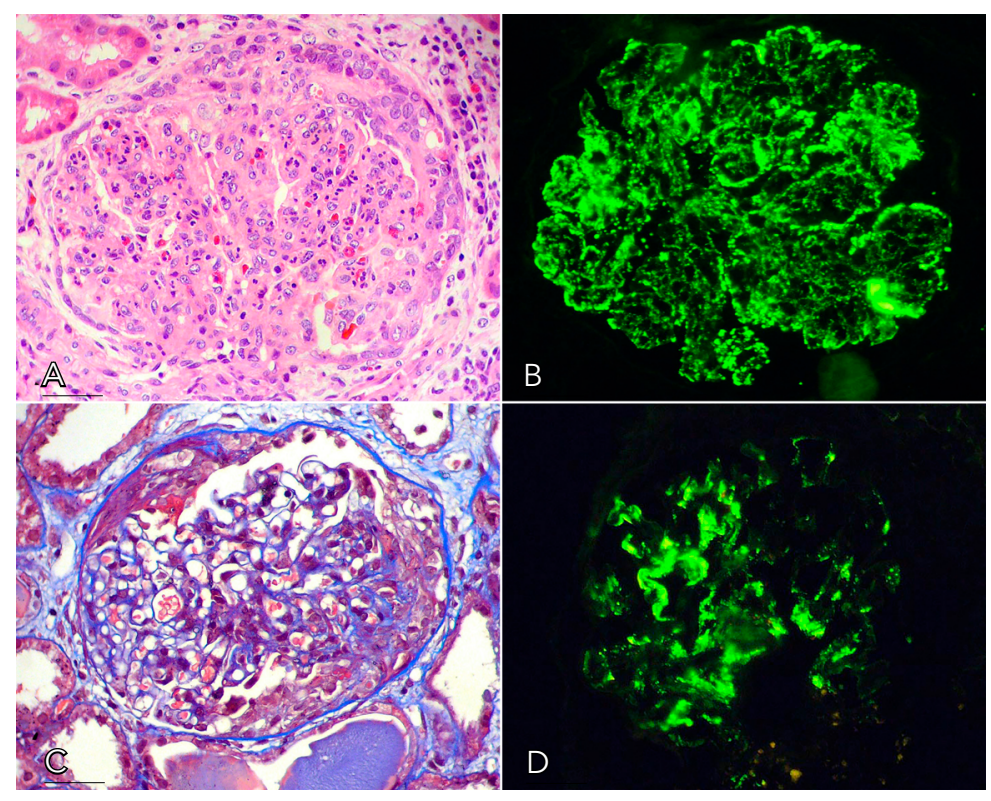

Figura 1. (A) Glomerulonefritis proliferativa endocapilar, postinfecciosa; además de la hipercelularidad endocapilar, hay semiluna epitelial (lado derecho del glomérulo). La mayoría de glomérulos presentaban proliferación extracapilar (hematoxilina y eosina, 400x). (B) Inmunofluorescencia directa para C3, donde se observan los gránulos variables en tamaño, muchos de ellos en las paredes capilares. Este aspecto es muy característico de la glomerulonefritis postinfecciosa (400x). (C) En este glomérulo de un paciente con nefropatía por IgA, hay dos semilunas epiteliales circunscritas, una en la parte superior izquierda y otra en el lado derecho (tricrómico de Masson, 400x). (D) Inmunofluorescencia directa para IgA en el mismo caso del panel $C$, donde se observa que además de la positividad mesangial, hay marcación en algunas paredes capilares, un hallazgo frecuente en los casos de nefropatía por lgA con proliferación extracapilar (400x).

go realizar la suspensión progresiva en los próximos 4 a 6 meses.

- Ciclofosfamida, la cual puede ser administrada de manera intravenosa o por vía oral de la siguiente manera:

- Intravenosa: 500 mg/m² a 1.000 $\mathrm{mg} / \mathrm{m}^{2}$ de superficie corporal, cada 15 días o cada mes, por 6 dosis (en un período de aproximadamente 3 a 6 meses, dependiendo de la etiología). En caso de compromiso grave de la función renal con necesidad de diálisis, se administra 50\% de la dosis.
- Oral: $2 \mathrm{mg} / \mathrm{kg}$ al día, por un período de 3 a 6 meses.

- Existen otras alternativas como el uso de rituximab, el cual ha sido analizado en varios estudios [3643], principalmente en tres etiologías de la GMNRP tipo II (nefritis lúpica, vasculitis por IgA y nefropatía por lgA), de los cuales se puede concluir que la terapia con este medicamento puede tener alguna utilidad, sobre todo cuando existe resistencia a los esteroides o cuando está contraindicado el uso de los medicamentos de primera 


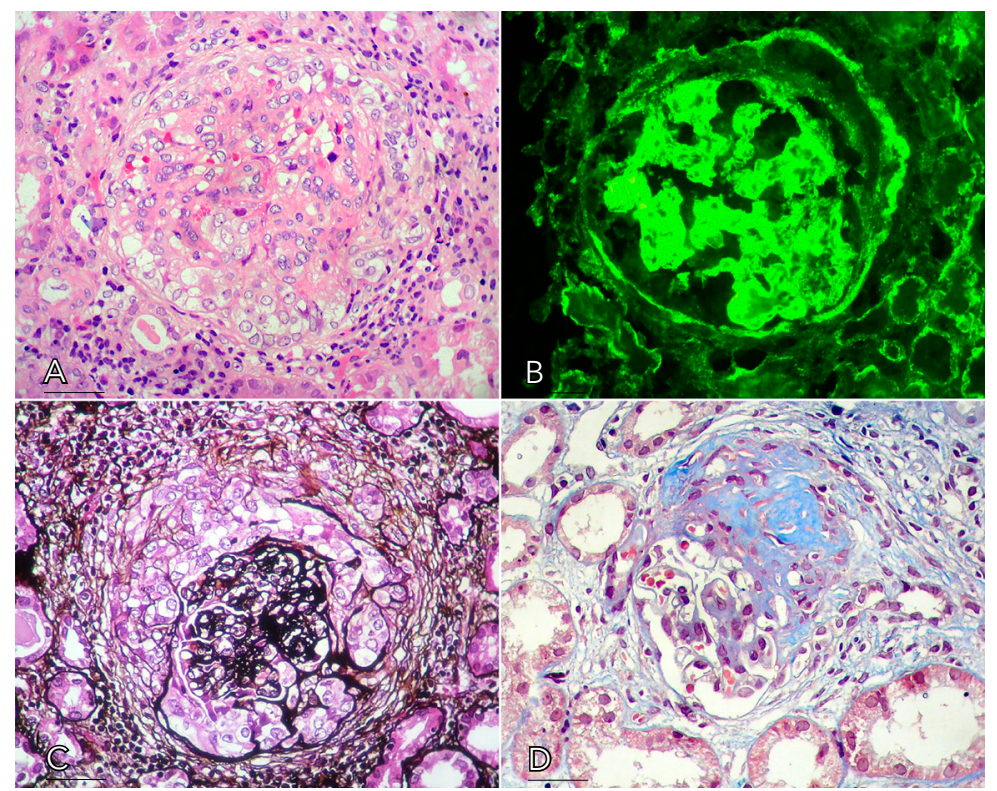

Figura 2. (A) Proliferación endo y extracapilar en una paciente con diagnóstico de nefritis lúpica, donde se observa la semiluna comprometiendo toda la circunferencia del espacio de Bowman (hematoxilina y eosina, 400x). (B) Inmunofluorescencia directa para IgG en el mismo caso del panel A; hay positividad parietal, mesangial y en basales tubulares y de cápsula de Bowman. En la parte superior derecha se insinúa una semiluna (400x). (C) En este glomérulo hay extensa destrucción de la cápsula de Bowman y ya se resaltan algunas fibras de colágeno (en negro) empezando a fibrosar la semiluna (plata-metenamina, 400x). (D) En este glomérulo de una paciente con nefropatía por IgA, la semiluna ha sido reemplazada por fibrosis (en azul): semiluna fibrosa, una lesión ya irreversible (tricrómico de Masson, 400x).

línea como la ciclofosfamida, sin embargo, los datos de los estudios son limitados y se requiere mayor investigación en este sentido. En la GMNRP tipo III, el rituximab sí está claramente aceptado como una alternativa de inducción con eficacia equivalente a la ciclofosfamida.

- En cuanto a la plasmaféresis, que tiene utilidad comprobada en la GMNRP tipo I y en menor grado en la tipo III, en el contexto de la tipo II, no parece tener tanta utilidad, con la excepción de aquellos pacientes que tengan hemorragia alveolar difusa, una enfermedad como un lupus grave refractario que ponga en peligro la vida, o como último recur- so en aquellos pacientes que no responden adecuadamente a la terapia de primera línea $[10,44]$.

Otro pilar fundamental del tratamiento de la GMNRP tipo II, es la identificación de la etiología específica y el tratamiento apropiado de la misma $[1,3,9]$, sin embargo, el objetivo del artículo no es una revisión profunda del tratamiento de cada etiología, por lo que solo se mencionará de manera general el tratamiento en algunas de las causas más comunes de la enfermedad:

- Nefropatía por IgA: la GMNRP asociada a nefropatía por IgA se trata con esteroides y ciclofosfamida, realizando el mantenimiento con 
azatioprina; una vez estabilizada la función renal, se recomienda el control de la proteinuria e hipertensión arterial con inhibidores de la enzima convertidora de angiotensina (IECA) o bloqueadores del receptor de angiotensina II (ARA II) $[45,46]$.

- Vasculitis por IgA: en el caso de GMNRP asociada a vasculitis por IgA, el tratamiento inmunosupresor es igual al descrito para la nefropatía por lgA; los pacientes se benefician además, del uso de medidas de soporte como hidratación, reposo y analgésicos [47].

- Nefritis lúpica con GMNRP: el pilar fundamental del tratamiento en estos pacientes es la terapia inmunosupresora agresiva con esteroides y ciclofosfamida, además, se debe ofrecer tratamiento para el control de la presión arterial, medidas antiproteinúricas, un antimalárico (cloroquina o hidroxicloroquina) y tratamiento de la dislipidemia (que también impacta en la proteinuria); esto contribuye también con el control de los factores de riesgo cardiovascular, muy importantes en estos pacientes [48,49].

- Glomerulonefritis postestreptocócica: la mayoría de estos pacientes tienen una resolución espontánea de la enfermedad, sin embargo, en algunos, sobre todo adultos, no se alcanza una respuesta completa, por lo que en esos casos se sugiere el uso de tratamiento inmunosupresor, aunque con poca evidencia $[50,51]$.

\section{Conclusión}

La GMNRP es un síndrome poco común pero extremadamente grave, caracterizado por el rápido deterioro de la función renal, asociado al daño de más del $50 \%$ de los glomérulos, lo cual es evidenciado en la histología por la formación de semilunas (proliferación extracapilar); un porcentaje importante de los casos es explicado por el depósito de complejos inmunes (GMNRP tipo (I), secundario a varias enfermedades, entre las que destacan la glomerulonefritis postestreptocócica, la nefropatía por lgA, la nefritis lúpica y la vasculitis por IgA, entre otras. Al identificar a los pacientes con esta patología de una manera oportuna, es posible iniciar un tratamiento adecuado y con ello mejorar el pronóstico, evitando la enfermedad renal crónica terminal y/o la muerte de los pacientes.

\section{Referencias}

1. Ramos JM, Medina AB, Fernández MP. Glomerulonefritis agudas y rápidamente progresivas. Medicine 2019;12:4717-4724. https://doi. org/10.1016/j.med.2019.05.029.

2. Couser WG. Rapidly progressive glomerulonephritis: classification, pathogenetic mechanisms, and therapy. Am J Kidney Dis 1988;11:449-464. https://doi.org/10.1016/ s0272-6386(88)80079-9.

3. Parmar MS, Bashir K. Crescentric Glomerulonephritis. StatPearls. Treasure Island (FL): StatPearls Publishing; 2021. Acceso 15 de abril de 2020. Disponible en https://www.ncbi.nlm.nih. gov/books/NBK430727/.

4. Sethi S, Haas M, Markowitz GS, D'Agati VD, Rennke HG, Jennette JC, et al. Mayo Clinic/ Renal Pathology Society consensus report on pathologic classification, diagnosis, and reporting of GN. J Am Soc Nephrol 2016;27:12781287. https://doi.org/10.1681/asn.2015060612.

5. Appel G, Radhakrishnan J. Enfermedades glomerulares y síndromes nefróticos. In: Goldman L, Schaefer A, eds. Tratado de Medicina Interna. Ámsterdam, Países Bajos: Elsevier; 2016. p. 783-793.

6. Johnson R, Feehally J, Floege J, Tonelli M. Introduction to glomerular disease : Clinical pre- 
sentations. Comprehensive Clinical Nephrology. Ámsterdam, Países Bajos: Elsevier; 2019. p. 184-198.

7. Praga-Terente M. Nefropatías glomerulares primarias. In: Rozman-Borstnar C, CardellachLópez F, eds. Medicina Interna. España: ACME libreria ciencia medicina; 2020. p. 845-854.

8. Baldwin DS, Neugarten J, Feiner HD, Gluck M, Spinowitz B. The existence of a protracted course in crescentic glomerulonephritis. Kidney Int 1987;31:790-794. https://doi.org/10.1038/ ki.1987.67.

9. Moroni G, Ponticelli C. Rapidly progressive crescentic glomerulonephritis: Early treatment is a must. Autoimmun Rev 2014;13:723-729. https://doi.org/10.1016/j.autrev.2014.02.007.

10. Jennette JC. Rapidly progressive crescentic glomerulonephritis. Kidney Int 2003;63:1164-1177. https://doi.org/10.1046/j.1523-1755.2003.00843.x.

11. Choudhury TA, Singh RG, Singh $S$, Singh TB, Rathore SS. Clinicopathologic spectrum of crescentic glomerulonephritis: a hospital-based study. Saudi J Kidney Dis Transpl 2014;25:689-696. https://doi.org/10.4103/1319-2442.132241.

12. Kidney Disease: Improving Global Outcomes (KDIGO). Chapter 10: Immunoglobulin A nephropathy. Kidney Int Suppl (2011) 2012;2:209-217. https://doi.org/10.1038/kisup.2012.23.

13. Aguiar A, Alvarer $P$, Paz-Wasiuchnik V, Abib A, Ciliberti E, Moretti L, et al. Glomerulonefritis rápidamente progresiva en ancianos. Medicina (Buenos Aires) 2013;73:148-152.

14. Wu T, Peng J, Meng T, Liu $Q$, Ao $X$, Lin W, et al. Clinicopathological features and prognostic analysis of 49 cases with crescentic glomerulonephritis. Exp Ther Med 2019;18:3984-3990. https://doi.org/10.3892/etm.2019.8023.

15. Chen M, Cui Z, Zhao MH. ANCA-associated vasculitis and anti-GBM disease: the experience in China. Nephrol Dial Transplant 2010;25:2062-2065. https://doi.org/10.1093/ ndt/gfq134.

16. Bonsib SM. Glomerular basement membrane discontinuities. Scanning electron microscopic study of acellular glomeruli. Am J Pathol 1985; 119:357-360.

17. Atkins RC, Nikolic-Paterson DJ, Song $\mathbf{Q}$, Lan HY. Modulators of crescentic glomerulone- phritis. J Am Soc Nephrol 1996;7:2271-2278.

https://doi.org/10.1681/asn.V7112271.

18. Praga M, Gutierrez-Millet V, Navas JJ, Ruilope LM, Morales JM, Alcazar JM, et al. Acute worsening of renal function during episodes of macroscopic hematuria in $\lg A$ nephropathy. Kidney Int 1985;28:69-74. https://doi. org/10.1038/ki.1985.120.

19. Bennett WM, Kincaid-Smith P. Macroscopic hematuria in mesangial IgA nephropathy: correlation with glomerular crescents and renal dysfunction. Kidney Int 1983;23:393-400. https://doi.org/10.1038/ki.1983.32.

20. Arimura $Y$, Muso E, Fujimoto $S$, Hasegawa M, Kaname S, Usui J, et al. Evidence-based clinical practice guidelines for rapidly progressive glomerulonephritis 2014. Clin Exp Nephrol 2016;20:322-341. https://doi.org/10.1007/ s10157-015-1218-8.

21. Bruns FJ, Adler S, Fraley DS, Segel DP. Long term follow-up of aggressively treated idiopathic rapidly progressive glomerulonephritis. Am J Med 1989;86:400-406. https://doi. org/10.1016/0002-9343(89)90336-7.

22. Bagga A, Menon S. Rapidly progressive glomerulonephritis. In: Geary D, Schaefer F, eds. Comprehensive Pediatric Nephrology Mosby. 1st ed; 2008. p. 319-328.

23. Rodríguez-Iturbe $\mathbf{B}$. Epidemic poststreptococcal glomerulonephritis. Kidney Int 1984;25:129136. https://doi.org/10.1038/ki.1984.19.

24. Nissenson AR, Baraff LJ, Fine RN, Knutson DW. Poststreptococcal acute glomerulonephritis: fact and controversy. Ann Intern Med 1979;91:76-86. https://doi.org/10.7326/00034819-91-1-76.

25. Blyth CC, Robertson PW, Rosenberg AR. Post-streptococcal glomerulonephritis in Sydney: a 16-year retrospective review. J Paediatr Child Health 2007;43:446-450. https://doi. org/10.1111/j.1440-1754.2007.01109.x.

26. Sanjad S, Tolaymat A, Whitworth J, Levin S. Acute glomerulonephritis in children: a review of 153 cases. South Med J 1977;70:12021206. https://doi.org/10.1097/00007611 197710000-00015.

27. Sagel I, Treser G, Ty A, Yoshizawa N, Kleinberger $\mathbf{H}$, Yuceoglu AM, et al. Occurrence and nature of glomerular lesions after group 
A streptococci infections in children. Ann Intern Med 1973;79:492-499. https://doi. org/10.7326/0003-4819-79-4-492.

28. Gutiérrez E, González $E$, Hernández E, Morales E, Martínez MA, Usera G, et al. Factors that determine an incomplete recovery of renal function in macrohematuria-induced acute renal failure of IgA nephropathy. Clin J Am Soc Nephrol 2007;2:51-57. https://doi. org/10.2215/cjn.02670706.

29. Peru H, Soylemezoglu O, Bakkaloglu SA, Elmas S, Bozkaya D, Elmaci AM, et al. Henoch Schonlein purpura in childhood: clinical analysis of 254 cases over a 3-year period. Clin Rheumatol 2008;27:1087-1092. https://doi. org/10.1007/s10067-008-0868-2.

30. Trapani S, Micheli A, Grisolia F, Resti M, Chiappini E, Falcini F, et al. Henoch Schonlein purpura in childhood: epidemiological and clinical analysis of 150 cases over a 5-year period and review of literature. Semin Arthritis Rheum 2005;35:143-153. https://doi.org/10.1016/j.semarthrit.2005.08.007.

31. Peeters V, De Raeve L. Blistering eruptions in Henoch-Schönlein syndrome: more common than assumed. Eur J Pediatr 2018;177:475-476. https://doi.org/10.1007/s00431-017-2990-0.

32. Wu CS, Tung SY. Henoch-Schönlein purpura complicated by upper gastrointestinal bleeding with an unusual endoscopic picture. J Clin Gastroenterol 1994;19:128-131. https://doi. org/10.1097/00004836-199409000-00011.

33. Nathan K, Gunasekaran TS, Berman JH. Recurrent gastrointestinal Henoch-Schönlein purpura. J Clin Gastroenterol 1999;29:86-89. https:// doi.org/10.1097/00004836-199907000-00022.

34. Johnson EF, Lehman JS, Wetter DA, Lohse CM, Tollefson MM. Henoch-Schönlein purpura and systemic disease in children: retrospective study of clinical findings, histopathology and direct immunofluorescence in 34 paediatric patients. Br J Dermatol 2015;172:1358-1363. https://doi.org/10.1111/bjd.13472.

35. Greenhall GH, Salama AD. What is new in the management of rapidly progressive glomerulonephritis? Clin Kidney J 2015;8:143-150. https://doi.org/10.1093/ckj/sfv008.

36. Rovin BH, Furie R, Latinis $K$, Looney RJ, Fervenza FC, Sanchez-Guerrero J, et al. Efficacy and safety of rituximab in patients with active proliferative lupus nephritis: the Lupus $\mathrm{Ne}$ phritis Assessment with Rituximab study. Arthritis Rheum 2012;64:1215-1226. https://doi. org/10.1002/art.34359.

37. Condon MB, Ashby D, Pepper RJ, Cook HT, Levy JB, Griffith M, et al. Prospective observational single-centre cohort study to evaluate the effectiveness of treating lupus nephritis with rituximab and mycophenolate mofetil but no oral steroids. Ann Rheum Dis 2013;72:1280-1286. https://doi.org/10.1136/ annrheumdis-2012-202844.

38. Ramos-Casals M, Diaz-Lagares $C$, SotoCardenas MJ, Brito-Zeron P, Cuadrado MJ, Sanna G, et al. Rituximab therapy in lupus nephritis: current clinical evidence. Clin Rev Allergy Immunol 2011;40:159-169. https://doi. org/10.1007/s12016-010-8205-3.

39. Tsanian M, Solov'ev SK, Torgashina AV, Aleksandrova EN, Radenska-Lopovok SG, Nikolaeva EV, et al. [Long-term follow-up of patients with refractory systemic lupus erythematosus during rituximab treatment]. Ter Arkh 2014;86:40-49.

40. Lafayette RA, Canetta PA, Rovin BH, Appel GB, Novak J, Nath KA, et al. A randomized, controlled trial of rituximab in IgA nephropathy with proteinuria and renal dysfunction. J Am Soc Nephrol 2017;28:1306-1313. https://doi. org/10.1681/asn.2016060640.

41. El-Husseini A, Ahmed A, Sabucedo A, FabuIo E. Refractory Henoch-Schönlein purpura: atypical aetiology and management. J Ren Care 2013;39:77-81. https://doi.org/10.1111/ j.1755-6686.2013.12007.x.

42. Fenoglio R, Naretto C, Basolo B, Quattrocchio G, Ferro $\mathbf{M}$, Mesiano $\mathbf{P}$, et al. Rituximab therapy for IgA-vasculitis with nephritis: a case series and review of the literature. Immunol Res 2017;65:186-192. https://doi.org/10.1007/ s12026-016-8827-5.

43. Chancharoenthana $W$, Townamchai $N$, Leelahavanichkul A, Wattanatorn S, Kanjanabuch T, Avihingsanon $\mathbf{Y}$, et al. Rituximab for recurrent IgA nephropathy in kidney transplantation: A report of three cases and proposed mechanisms. Nephrology (Carlton) 2017;22:65-71. https://doi.org/10.1111/nep.12722. 
44. Chen $\mathbf{X}$, Chen N. Plasma exchange in the treatment of rapidly progressive glomerulonephritis. Contrib Nephrol 2013;181:240-247. https://doi.org/10.1159/000348632.

45. Appel GB, Waldman M. The IgA nephropathy treatment dilemma. Kidney Int 2006;69:19391944. https://doi.org/10.1038/sj.ki.5000434.

46. Barratt J, Feehally J. Treatment of IgA nephropathy. Kidney Int 2006;69:1934-1938. https:// doi.org/10.1038/sj.ki.5000419.

47. Saulsbury FT. Henoch-Schönlein purpura in children. Report of 100 patients and review of the literature. Medicine (Baltimore) 1999;78:395-409. https://doi. org/10.1097/00005792-199911000-00005.

48. Cheigh JS, Kim H, Stenzel KH, Tapia L, Sullivan JF, Stubenbord W, et al. Systemic lupus erythematosus in patients with end-stage renal disease: long-term follow-up on the prognosis of patients and the evolution of lupus activity. Am J Kidney Dis 1990;16:189-195. https://doi. org/10.1016/s0272-6386(12)81017-1.

49. Chagnac A, Kiberd BA, Fariñas MC, Strober S, Sibley RK, Hoppe R, et al. Outcome of the acute glomerular injury in proliferative lupus nephritis. J Clin Invest 1989;84:922-930. https://doi.org/10.1172/jci114254.

50. Raff A, Hebert T, Pullman J, Coco M. Crescentic post-streptococcal glomerulonephritis with nephrotic syndrome in the adult: is aggressive therapy warranted? Clin Nephrol 2005;63:375380. https://doi.org/10.5414/cnp63375.

51. Roy S 3rd, Murphy WM, Arant BS Jr. Poststreptococcal crescenteric glomerulonephritis in children: comparison of quintuple therapy versus supportive care. J Pediatr 1981;98:403-410. https://doi.org/10.1016/s0022-3476(81)80703-2. 